ORIGINAL ARTICLE

\title{
Quantitative analysis of Protective Role of salivary protein on demineralization of enamel
}

\author{
ASADULLAH RATHORE ${ }^{1}$, ANWAR ALAM ${ }^{2}$, MARRYAM RIAZ ${ }^{3}$, ASMA ARSHAD ${ }^{4}$, MARIA JAVED 5 , FARIHA FAYYAZ ${ }^{6}$, \\ SANA CHAUDHRY7 \\ ${ }^{1}$ Assistant Professor Oral Biology, Azra Naheed Dental College, Superior University, Lahore. \\ ${ }^{2}$ Associate Professor Oral Biology, Azra Naheed Dental College, Superior University, Lahore. \\ ${ }^{3}$ Assistant Professor Physiology, Azra Naheed Dental College, Superior University, Lahore. \\ ${ }^{4}$ Assistant Professor Biochemistry, Azra Naheed Dental College, Superior University, Lahore. \\ ${ }^{5}$ Associate Professor Oral Pathology, Azra Naheed Dental College, Superior University, Lahore. \\ ${ }^{6}$ Assistant Professor Community dentistry, Azra Naheed Dental College, Superior University, Lahore. \\ ${ }^{7}$ Associate Professor, Aviecenna Dental College, Lahore \\ Correspondence to: Dr. Asadullah Rathore, Email: asadullah_rath@hotmail.com
}

\begin{abstract}
Objective: The aim of this study was to analyze the effect of salivary proteins (statherin and histatin-1) on demineralized enamel surface and to study changes in texture, magnitude and direction of crystallites and changes in prismatic structure of enamel respectively.

Material \& Methods: Synchrotron x-ray diffraction technique to determine the variation in degree of crystal orientation (texture). Incisors were demineralized and sectioned to 300-500 microns, rinsed with salivary protein solutions of statherin and histatin separately and in combination with short and full-lengths. A beam spot size of $20 \mu \mathrm{m} \times 20 \mu \mathrm{m}$ was used to obtain

2D diffraction patterns to distinguish orientation of crystallites.

Results: The contour maps as well as the SEM analysis present similar surface properties of the sample treated with STN-21 and the controlled PBS sample. Therefore, STN-21 was found potent in preventing demineralization and restoring surface enamel texture followed by STN-21+HTN-21 and STN43+HTN38. HTN-21 and HTN-38 showed similar demineralization

pattern as the controlled demineralized sample.

Conclusion: Ranking of demineralization among samples was found to be controlled demineralized $>\mathrm{HTN}-21=$ HTN-38 > STN-43+HTN-38 > STN-21+HTN-21 > STN-21. Developing STN-21 as a therapeutic against dental caries and erosion.
\end{abstract}

Keywords: Enamel, Demineralization, salivary proteins.

\section{INTRODUCTION}

Dental caries and erosion are two of the most common enamel pathologies which occur due to acid attack. However, the source of acid production in both cases differs as erosion results from acids in diet and source of carious activity are active bacteria. Caries is a thermodynamic process during which cavitation may occur once the crystal lattice is disorganized but under favorable conditions mineral content is gained by HAP crystallites leading to their growth to the natural length. ${ }^{1,2}$.

Saliva plays a vital role in maintenance and preservation of the oral hard and soft tissues. This salivary gland secretion has various constituents that make it multifunctional. ${ }^{3}$ Humphrey and Williamson discussed the potential antibacterial actions of salivary proteins, immunoglobulins and certain enzymes. Proteins as well as calcium and phosphate ions work in association to influence the dynamic process of demineralization and remineralization. ${ }^{3}$ Moreno et al. discussed how some proteins such proline-rich protein and statherin are involved in controlling the precipitation of calcium ions on powdered enamel. ${ }^{4}$ The precipitation and dissolution hydroxyapatite crystal is a controlled process regulated by either crystal growth within the crystal nuclei or due to the activity of salivary peptides that alter the HAP surface energy. ${ }^{5}$ Kosoric et al. studied the effectiveness of protein on HAP aggregation which also depends on degree of saturation, low HAP porosity and protein concentration. Hence found bovine albumin to be potent against demineralization. ${ }^{6}$ Kirkham et al. evidently supported the ability of peptides to induce mineral content to the enamel hydroxyapatite and also prevent surface demineralization. ${ }^{7}$

Aim of this study is to analyze the effect of salivary proteins (statherin and histatin-1) on demineralized enamel surface at nanoscale, with their full length and N-terminalmers (21) separately and in combination, using 2-D synchrotron X-ray diffraction and scanning electron microscope. The objective is to study changes in texture, magnitude and direction of crystallites and changes in prismatic structure of enamel respectively.

\section{MATERIAL \& METHODS}

2.1 Sample preparation: 7 incisors were first demineralized with acetic acid and then sectioned into slices of $300-500$ microns. 5 of these samples were exposed to statherin and histatin-1 separately and in combination with their truncated $\mathrm{N}$-terminals and full lengths, whereas 2 samples were kept in controlled group.

Sliced samples were wet-grounded with 100, 220, 320, 400 and 600-grit silicon carbide abrasive paper respectively (Buehler, IL, USA) kept on an automatic lapping and polishing machine (Kent 4, Kemet International Ltd., Maidstone, UK). Samples were then kept in distilled water containing multipurpose detergent and left for 10 minutes in an ultrasonic bath (Kerry PUL-125, Guyson International Ltd., North Yorkshire, UK). Fine particle size 
diamond polishing paste was used to polish the sample with sequence ranging from 6 microns to 0.25 microns (Met-Prep, Coventry, UK). Each sliced sample was further treated with $35 \%$ ortho-phosphoric acid for 15 seconds followed by dehydration is different ethanol concentrations i-e 25\%, 50\%, 75\%, 100\% respectively for 30 minutes and kept in desiccator for 2 days. Before placement into the chamber of SEM, tooth slices were coated with carbon.

\begin{tabular}{|l|l|}
\hline Controlled Group & Active Group \\
\hline 1.Rinse in phosphate buffer & Rinse with Statherin- \\
solution(PBS) & 21(STN-21) \\
2.Kept on PBS without exposure & With STN- \\
to further & $21+$ HTN21(histatin-21) \\
demineralization & With HTN-21 \\
& With HTN-38(full length) \\
& With STN-43+HTN-38 \\
\hline
\end{tabular}

2.2 Sample exposure to beamline (BM-28): Figure 2 show the specific area or a window of tooth section that was exposed to the synchrotron radiations. A wavelength of $0.82 \AA$ with a beam spot of $20 \mu \mathrm{m} \times 20 \mu \mathrm{m}$ was attained using vacuum slits and sample to detector distance was kept approximately $176 \mathrm{~mm}$.

2.3 Data analysis: Data collected in form of 2D diffraction patterns was analyzed using Fit 2D and Mofit software to attain composite images of all samples, saved as chi. files to view it as a picture. The first and second dimensions of array were kept as 2048, as these represent the number of pixels used by the 2D detector. The calibration of the powder diffraction data in the Fit2D software was done using Lanthanum Hexaboride (LaB6) diffraction pattern which helps to refine the values for the instrument parameters. In Fit2D the calibrants and lanthanum hexaboride commands were performed in order to refine the sample to detector distance and wavelength values. After this the azimuthal angle was determined from 0 to 360 degrees and outer and inner radius was marked around the 002 reflection. An intensity vs azimuthal angle plot fitted with Gaussian peak shape to fit the FWHM using Mofit software where sharp peaks indicated the preferred orientation of enamel and broad peaks show random distribution of crystallites.

\section{RESULTS}

The contour maps revealed by using sigma plot software present the variation in texture of all tooth sections These contour maps illustrate the texture distribution within a window of tooth section from surface towards the EDJ exposed to the synchrotron beamline. (Al-Jawad et al. mentioned the hierarchical organization of the enamel structure with graded texture distribution, as past research specifies the trend of high texture at the surface region that decreases as a function of depth close to the EDJ. ${ }^{8}$ Therefore, variation is such a trend is most likely due to demineralization with or without the presence of salivary protein. Such a texture variation can be observed in Figure $1 \mathrm{~A})$ the controlled sample that was rinsed in PBS, where FWHM is low near the surface representing high texture that cannot be observed near the EDJ as the FWHM is high. Figure $1 \mathrm{~B}$ ) show the demineralized controlled group that has low texture at the surface region for about 250 microns followed by a region of high texture region that tends decrease towards the EDJ. The sample treated with short-length statherin (STN-21) in figure $1 \mathrm{C}$ ) has low FWHM i-e around 40 degree, moving from the air-enamel interface up to 100-150 microns, hence showing higher surface texture similar to the controlled PBS sample with same characteristic trend near the EDJ. The figure 1 D) shows the contour map of sample exposed to combination of truncated statherin and histatin-1(STN-21+HTN-21). There is a narrow zone of low texture that increases after 50-100 microns in the sub-surface area. This shows that the peptide combination shows some resistance to demineralization but as effective as by STN-21. The trend of texture variation in samples treated with $\mathrm{HTN}-21$ and HTN-38 can be seen in figure 1E) and F) where there is similar high FWHM at the surface that decreases beyond the surface region but texture near the EDJ remains same as the other samples. Hence the ineffectiveness of these peptides on surface is evident. A clearly disrupted surface is also observed in figure $1 \mathrm{G}$ ) in which the sample was treated with combination of full length proteins i-e statherin and histatin. It shows slightly high texture on the surface in the first 200-250 microns that tends to decrease as seen from air-enamel interface into the enamel structure. Low texture is evident near the EDJ.

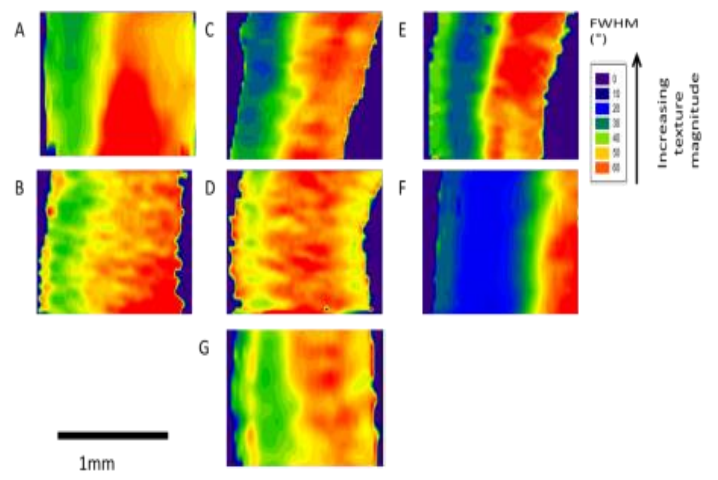

Figure 1. Presents texture distribution of A) controlled PBS sample. B) Sample rinsed with STN-21. C) Controlled demineralized, D) sample rinsed with STN21+HTN-21, E) Rinsed with HTN-21, F) Rinsed with HTN-38, G) Sample rinsed with STN-43+HTN-38.

3.1 SEM Results: The surface analysis of samples under the scanning electron microscope reveals that the controlled PBS sample in figure $2 \mathrm{H}$ ) has a smooth surface as the sample has not been demineralized. Therefore, prism structure is visible near the surface and even in the bulk enamel. On contrary, the controlled demineralized sample has a disrupted surface, indicating the effected of acid etching of the surface and also in the bulk region as evident in figure 2I).

The prism core seems more effected as compared to the periphery. The figure $2 \mathrm{~J}$ ) shows the image of sample treated with STN-21 that corresponds to the controlled group sample in which the surface is regular. The subsurface is etched as a result of demineralization but the surface seems to be restored due the effect of peptide exposure. Minor irregularities are visible on sample that was rinsed with STN-21+HTN-21, that could be due to some resistance shown by this protein combination in 
figure $2 \mathrm{~K})$. However, the effect is not as good as of STN21. The surface irregularities as well as dissolved prism core in bulk region of the circular arranged prism in figure showing HTN-21 treated sample. Figure 2L) defines similar projections seen in figure $2 \mathrm{l}$ ) and $\mathrm{K}$. This sample as rinsed with HTN-38 and the surface analysis appears to be irregular with enough evidence of this solution not being potent to prevent acid attack. The same features can be observed in the sample rinsed with combination of STN$43+\mathrm{HTN}-38$ as the etched surface and dissolved prism core is visible in the bulk enamel.

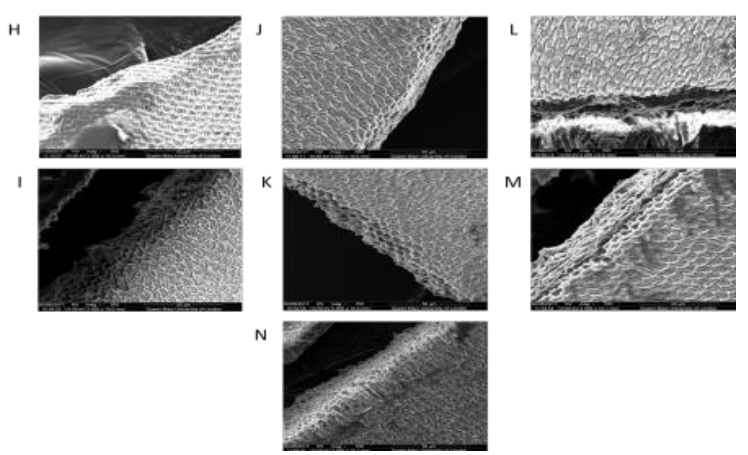

Figure 2 Shows variation in prism structure when exposed to different environments, where image $H$ ) is of controlled PBS sample. I) controlled demineralized. Image $(\mathrm{J}-\mathrm{N})$ were rinsed with STN-21, STN-21+HTN-21, HTN-21, HTN-38 and STN-43+HTN-38 respectively.

\section{DISCUSSION}

The synchrotron X-ray diffraction describes the arrangement of crystallites within the different regions of enamel. It is known from the past studies through synchrotron analysis that high texture is present near the enamel surface that decreases as a function of depth.8-10

The current study illustrates the effect of salivary proteins on demineralized enamel under SEM and synchrotron analysis. Variation in full width half maximum (FWHM) describes the texture variation in contour maps of all samples. It known from the results that tooth section rinsed with truncated statherin (STN-21) has higher texture at the surface. Its similarities are evident with the surface texture of controlled PBS sample that can be seen in texture distribution maps as well as the SEM images. This supports the precious scanning micro-radiograph (SMR) studies that suggest STN-15 or more to be a potent peptide in preventing mineral loss and restoration of the demineralized enamel section. ${ }^{11}$ Kosoric et al. also found STN-21 to be effective in preventing demineralization of an artificial erosive lesion. ${ }^{12}$ This study also substantiates the past research on the adsorptive behavior of synthetic statherin on hydrophobic and hydrophilic surfaces. Santos et al. suggested similar adsorption ability of statherin and STN-21 on HAP as compared to the hydrophobic silica surface, besides weaker bonding was observed on the hydrophilic silica. ${ }^{13}$ The surface of sample rinsed with STN$21+\mathrm{HTN}-21$ shows resistance to certain extent that is less than the effectiveness of STN-21. Further low resistance to a few microns was observed in sample exposed to STN43+HTN-38 solution. The phenomena can also be observed in the SEM images where slight projection on the surface presents a rough appearance that is not the case in controlled PBS and the STN-21 treated sample. On the other hand, samples that were rinsed with full length and truncated histatin displays a low textured on the surface in the contour maps whereas, surface irregularities and etched enamel prism core can also be seen. Such a demineralization in form of low surface texture and etched enamel prism structure is also prominent in the controlled demineralized sample. This similarity specifies the inability of histatin peptides in prevention and restoration of enamel surface texture in comparison to the truncated statherin (STN-21). Hence, the sequence of samples from most to the least demineralized was observed as: controlled demineralized $>\mathrm{HTN}-21=\mathrm{HTN}-38>\mathrm{STN}-43+\mathrm{HTN}-38>$ STN-21+HTN-21 > STN-21.

\section{CONCLUSION}

The synchrotron radiations provide a precise insight of the textural changes within the enamel as a function of depth. The magnitude and direction of crystallites signifies the changes in texture in different regions of tooth. The results of this study relate well to the previous SMR studies, that detects the percentage mineral loss. The SEM is a qualitative analysis that makes its comparison difficult with the synchrotron study, yet the past SMR analysis is well supported by this study.

\section{REFERENCES}

1. Abou Neel EA, Aljabo A, Strange A, Ibrahim S, Coathup M, Young AM, Bozec L, Mudera V. Demineralization-remineralization dynamics in teeth and bone. Int J Nanomedicine. 2016;11:4743-4763.

2. Anderson P, Kosoric J, Hector MP. The "calcium management" action of salivary proteins forcaries and erosion prevention. CPD Dent. 2007;6:31-37

3. Humphrey SP, Williamson RT. A review of saliva: normal composition, flow, and function. J Prosthet Dent. 2001;85(2):162-9.

4. Moreno EC, Varughese K, Hay DI. Effect of human salivary proteins on the precipitation kinetics of calcium phosphate. Calcif Tissue Int. 1979;28(1):7-16.

5. Tang R, Hass M, Wu W, Gulde S, Nancollas GH. Constant composition dissolution of mixed phases. II. Selective dissolution of calcium phosphates. J Colloid Interface Sci. 2003;260(2):379-84.

6. Kosoric J, Hector MP, Anderson P. The influence of proteins on demineralization kinetics of hydroxyapatite aggregates. J Biomed Mater Res A. 2010;94(3):972-7.

7. Kirkham J, Firth A, Vernals D, Boden N, Robinson C, Shore RC, et al. Self-assembling peptide scaffolds promote enamel remineralization. J Dent Res. 2007;86(5):426-30.

8. Al-Jawad M, Steuwer A, Kilcoyne SH, Shore RC, Cywinski R, Wood DJ. 2D mapping of texture and lattice parameters of dental enamel. Biomaterials. 2007;28(18):2908-14.

9. Al-Jawad M, Addison $O$, Khan MA, James A, Hendriksz CJ. Disruption of enamel crystal formation quantified by synchrotron microdiffraction. J Dent. 2012;40(12):1074-80.

10. Yagi N, Ohta N, Matsuo T, Tanaka T, Terada Y, Kamasaka H, et al. Evaluation of enamel crystallites in subsurface lesion by microbeam X-ray diffraction. J Synchrotron Radiat. 2009;16(Pt 3):398-404.

11. Shah S, Kosoric J, Hector MP, Anderson P. An in vitro scanning microradiography study of the reduction in hydroxyapatite demineralization rate by statherin-like peptides as a function of increasing N-terminal length. Eur J Oral Sci. 2011;119 Suppl 1:13-8.

12. Kosoric J, Williams RA, Hector MP, Anderson P. A synthetic peptide based on a natural salivary protein reduces demineralisation in model systems for dental caries and erosion. Int J Peptide Res Therapeut. 2007;13(4):497-503.

13. Santos O, Kosoric J, Hector MP, Anderson P, Lindh L. Adsorption behavior of statherin and a statherin peptide onto hydroxyapatite and silica surfaces by in situ ellipsometry. J Colloid Interface Sci. 2008;318(2):175-82. 